\title{
Kinetic ballooning modes in tokamaks and stellarators
}

\author{
K. Aleynikova ${ }^{1,2}$, A. Zocco ${ }^{1}$, P. Xanthopoulos ${ }^{1}$, P. Helander ${ }^{1}$, C. Nührenberg ${ }^{1}$ \\ ${ }^{1}$ Max-Planck-Institut für Plasmaphysik, \\ EURATOM Association, Greifswald, Germany and \\ ${ }^{2}$ Moscow Institute of Physics and Technology, Dolgoprudny, Russian Federation
}

\begin{abstract}
Kinetic ballooning modes (KBMs) are investigated by means of linear electromagnetic gyrokinetic (GK) simulations in the stellarator Wendelstein 7-X (W7-X), for high- $\beta$ plasmas, where $\beta$ is the ratio of thermal to magnetic plasma pressure. The analysis shows suppression of ion-temperaturegradient (ITG) and trapped particle (TEM) modes by finite- $\beta$ effects and destabilization of KBMs at high $\beta$. The results are compared with a generic tokamak case.

We show that, for large pressure gradients, the frequency of KBMs evaluated by the GENE code is in agreement with the analytical prediction of the diamagnetic modification of the ideal magnetohydrodynamic limit in W7-X general geometry. Thresholds for destabilization of the KBM are predicted for different W7-X equilibrium configurations. We discuss the relation of these thresholds to the ideal MHD stability properties of the corresponding equilibria.
\end{abstract}




\section{INTRODUCTION}

In a fusion reactor plasma, high density and temperature are necessary to achieve favourable reaction conditions. This implies high plasma $\beta$ s: the ratio of thermal to magnetic pressure. However, under such conditions, the curvature of the confining magnetic field and the plasma pressure gradient can excite kinetic ballooning modes (KBMs). The study of electromagnetic micro-turbulence due to KBMs is a topic which is largely unexplored in stellarator geometry and in particular for the stellarator Wendelstein 7-X (W7-X).

Other instabilities are of course also relevant in W7-X. The electrostatic ion-temperaturegradient-driven (ITG) and trapped-electron (TEM) modes have been extensively studied by means of gyrokinetic simulations [1-7], but KBMs have been explored less in stellarators than in tokamaks [8-12]. KBMs have recently been studied recently in helical plasmas [13], for a model configuration of standard LHD plasmas. In the present work we present the first numerical study of KBMs in W7-X. Although global fully-gyrokinetic simulations of a large class of Alfvénic modes have been already performed for W7-X geometry [14], an extensive investigation of KBMs in W7-X is still missing.

In tokamaks, numerical simulations show that the threshold for KBM destabilization is often lower than that corresponding to the ideal MHD ballooning mode $[15,16]$. In a recent work [17], it was shown that, at long-wavelengths, a finite- $\beta$ regime of KBMs can be adequately described by a simple diamagnetic modification of the ideal MHD equation as long as local magnetic drifts are kept consistent with the equilibrium constraint $\mathbf{j} \times \mathbf{B}=\nabla p$. However, this result was only demonstrated for a simple $\hat{s}-\alpha$ equilibrium model [18] and needs to be extended to more realistic geometry.

In this article we investigate KBMs in $\mathrm{W} 7-\mathrm{X}$ and we compare the behaviour of this instability to a generic tokamak case. Our study also includes the electromagnetic versions of other microinstabilities such as the ITG and TEM. The influence of parallel magnetic fluctuations on the destabilisation of KBM is also considered, while this aspect has been neglected in many previous numerical studies. Since the effect of plasma compressibility is closely related to the equilibrium pressure gradient [19], we always use gyrokinetic input quantities (pressure gradient and plasma $\beta$ ) consistent with the considered equilibria.

This work is structured in the following way: in Section II we summarise the relevant results of the general KBM theory of Ref. [16], introduce the simplified KBM equation of 
Ref. [17] for the tokamak case and discuss necessary modifications connected with stellarator geometry. In Section III we present results of linear electromagnetic GENE [20, 21] calculations of the KBM threshold for destabilization in different W7-X configurations. We also compare the phenomenology of ITG and TEMs in tokamak and W7-X for finite $\beta$. Our conclusions are discussed in Section IV.

\section{HIGH- $\beta$ ORDERING FOR KBMS EQUATION}

The basic theory of kinetic ballooning modes was developed in Ref. [16]. Here, the authors solve the gyrokinetic equation by expanding in $\varepsilon=v_{t h i}^{2} / \omega^{2} l_{c}^{2} \ll 1$, where $v_{t h i}=\sqrt{2 T_{i} / m_{i}}$ is the ion thermal speed, $l_{c}$ the connection length and $\omega \ll v_{t h e} / l_{c}$ is the mode frequency, with $v_{\text {the }}$ the electron thermal speed. The general KBM equation below retains magnetic drift resonances, gyro-averaging and magnetic compressibility effects, but trapped particles are neglected. The result is a second order differential equation for the electrostatic potential $\phi$ that reads:

$$
\frac{1}{\beta_{i} B} \frac{v_{t h s}^{2} / l_{c}^{2}}{\omega^{2}} \frac{\partial}{\partial z} b B \frac{\partial \phi}{\partial z}=K \phi
$$

with

$$
\begin{aligned}
K & =\left\{\left[Q-\left(1-\frac{\omega_{* i}}{\omega}\right)\right]\left[\alpha_{0, e}\left(1+\frac{\beta_{i}}{2} R\right)-\alpha_{1, e} \tau Q^{\prime} \frac{\beta_{i}}{2}\right]\right. \\
& \left.-\frac{\beta_{i}}{2}\left(Q^{\prime}+\alpha_{1, e}\right)\left[\alpha_{0, e} Q^{\prime}+\alpha_{1, e}(1+\tau-\tau Q)\right]\right\} \times\left\{(1+\tau-\tau Q)\left(1+\frac{\beta_{i}}{2} R\right)+\tau Q^{\prime 2} \frac{\beta_{i}}{2}\right\}^{-1} \\
& -\alpha_{1, e} \frac{\omega_{\kappa}+\omega_{B}}{\omega}
\end{aligned}
$$

where we defined the connection length $l_{c}$ and the coordinate $z$ along the field is defined so that $l_{c} \nabla_{\|}=\partial_{z}, \beta_{i}=8 \pi p_{i} / B_{a}^{2}, \quad b=k_{\perp}^{2} v_{t h i}^{2} / 2 \Omega_{i}^{2} B, \mathbf{k}_{\perp}$ is the wavevector across the equilibrium magnetic field, $\Omega_{i}(B)=m_{i} c /(e B)$ is the ion cyclotron frequency, $\alpha_{n, j}=1-\left(\omega_{* i} / \omega\right)\left(1+n \eta_{j}\right), \tau=T_{e} / T_{i}, \eta_{i}=L_{n_{i}} / L_{T_{i}}, \omega_{* i, e}=\frac{1}{2} k_{y} \rho_{i, e} v_{t h} / L_{n}, \omega_{B}=$ $\left(\mathbf{k}_{\perp} \rho_{s} / 2\right) \cdot v_{t h s} \hat{\mathbf{b}} \times \nabla B / B, \omega_{\kappa}=\left(\mathbf{k}_{\perp} \rho_{s} / 2\right) \cdot v_{t h s} \hat{\mathbf{b}} \times(\hat{\mathbf{b}} \cdot \nabla \hat{\mathbf{b}}), \rho_{s}=v_{t h s} / \Omega_{s}\left(B_{a}\right)$, where $B_{a}$ is a reference constant magnetic field, $L_{T i, e}$ and $L_{n}$ are the characteristic gradient lengths for temperature and density and $Q, Q^{\prime}, R$ are velocity-space integrals (defined in [16]). 
In the recently published work [17] the problem of kinetic ballooning mode instability was revisited in simple tokamak geometry. The authors derived an appropriate $\beta$-ordering, which allows Eq. (2) to be simplified greatly. The results of the kinetic instability analysis and its comparison with numerics lead to a natural distinction between "high-temperaturegradient" and "moderate-temperature-gradient" KBM regimes.

In the first case, instability occurs only for mode frequencies such that $\omega_{r}=\omega_{p i} / 2$, where $\omega_{p i}=\omega_{* i}\left(1+\eta_{i}\right)$. The maximum growth rate is located at very long wavelength. If magnetic drifts are kept consistent with the equilibrium pressure gradient, the gyrokinetic codes GS2 $[22,23]$ and GENE $[20,21]$ show excellent quantitative agreement with the familiar idealMHD equation with a diamagnetic correction [24]:

$$
\frac{1}{\beta_{i}} \frac{v_{t h i}^{2}}{\omega^{2} l_{c}^{2}} \frac{\partial}{\partial z} b B \frac{\partial \phi}{\partial z}=-\frac{2 \omega_{\kappa} \omega_{p}}{\omega^{2}} \phi-b\left[1-\frac{\omega_{* i}}{\omega}\left(1+\eta_{i}\right)\right] \phi,
$$

where $\omega_{p}=\omega_{* i}\left(1+\eta_{i}\right)-\omega_{* e}\left(1+\eta_{e}\right) \equiv \omega_{p i}+\omega_{p e}$ and $\omega_{\kappa} \neq \omega_{B}$ since $\beta$ is finite. Keeping $\omega_{\kappa}=\omega_{B}$ does not give a satisfactory agreement between analytics and numerics.

In more complicated geometries, Eq.(1) is still valid. In order to relate KBM theory to stellarator equilibrium codes, it is useful to introduce a modification of Boozer coordinates [25], that respects the field alignment:

$$
\left(x^{1}, x^{2}, x^{3}\right)=\left(s, q(s)\left(\theta-\theta_{0}\right)-\zeta, \theta-\theta_{0}\right),
$$

where $s=\Phi / \Phi_{\text {edge }}$, with $\Phi$ the toroidal magnetic flux and $\Phi_{\text {edge }}$ its value at the last closed flux surface, and $\theta$ and $\zeta$ are the Boozer poloidal and toroidal angles, respectively, $q=\Phi^{\prime} / \Psi^{\prime}$, where $\Psi$ is the poloidal magnetic flux and $\theta_{0}$ is the free parameter of ballooning theory. Thus we obtain:

$$
\frac{1}{\beta_{i} \sqrt{g_{B}}} \frac{v_{t h i}^{2}}{\omega^{2} l_{c}^{2}} \frac{\partial}{\partial \theta} \frac{b}{\sqrt{g_{B}}} \frac{\partial \phi}{\partial \theta}=K \phi .
$$

Here $\sqrt{g_{B}}$ is the determinant of the Jacobian matrix, $K$ is described in Eq. (2), $a$ is a reference length scale, $\mathbf{k}_{\perp}^{2}=k_{i} k^{i}=k^{j} g_{j i} k^{i}$, and the $b$ term now becomes

$$
b=-\frac{1}{2} \frac{\rho_{i}^{2}}{a^{2}} \frac{B_{a}^{2}}{B^{2}} \frac{1}{\sqrt{g_{B}}} \sum_{i, j=1}^{2} \frac{\partial}{\partial x^{i}} \sqrt{g_{B}} g^{i j} \frac{\partial}{\partial x^{j}} .
$$

Therefore, the magnetic geometry enters the KBM equation for stellarators through the magnetic field $B$, the Jacobian and through the $\mathbf{k}_{\perp}$ terms. In the framework of a flux tube approach, all of these quantities are functions of the field-line following coordinate. 
While in a stellarator one could expect a rather complicated outcome from the numerical solution of Eq.(4), but in fact we will see that it shares some fundamental features with the "high temperature-gradient" KBM solution of the tokamak case of Ref.[17].

\section{NUMERICAL RESULTS FOR W7-X}

In the present section, we first describe the main parameters of the magnetic configurations that will be considered in this linear study, namely the stellarator W7-X and a standard tokamak, both in the context of the flux tube approach. Numerical simulation results are then presented for various sets of physical parameters.

\subsection{Simulation details}

The gyrokinetic simulations are carried out with the GENE code, which uses input data from the VMEC [26] and GIST [27] codes for data concerning the geometry of the magnetic field. The simulations are carried out for a range of density $\left(a / L_{n i, e}\right)$ and temperature $\left(a / L_{T i, e}\right)$ gradients, where $a$ is a normalisation length, and as already mentioned, it is important to vary the magnetic equilibrium from simulation to simulation so that it is consistent with the resulting pressure gradient. The magnetic equilibrium is varied in a following way: we keep the shape of the pressure profile constant but vary its absolute magnitude, so that $\beta$ becomes proportional to the pressure gradient.

We consider a hydrogen plasma in the collisionless regime, in the W7-X (KJM and TEH configurations, two tubes: one with its center in the outboard midplane of the so-called beanshaped plane, the other centred around the outboard midplane of the "triangular" plane, both tubes extend one poloidal turn around the torus) and generic tokamak geometry, with electromagnetic effects and consistent pressure gradients, therefore: $T_{i} / T_{e}=1, m_{i} / m_{e}=$ 1836, $r / R=0.26, q=1.3$, local magnetic shear $\hat{s}=0.745$ for the tokamak case and $r / R \approx 0.095, q=1.1, \hat{s} \approx-0.1$ for the W7-X case. Here, $T_{i, e}$ are the ion and electron temperature, $R$ is the major radius, $r$ is the minor radius and $\hat{s}$ is the global magnetic shear. Here, $\beta_{G E N E}=\beta_{i, e}=\frac{\beta_{t o t a l}}{2}=\frac{8 \pi n_{i 0} T_{r e f}}{B_{r e f}^{2}}$, where $T_{\text {ref }}$ is a reference temperature, $B_{\text {ref }}$ a reference magnetic field and $n_{i_{0}}$ is the equilibrium ion density.

We consider $k_{x} \rho_{s}=0$, where $k_{x}$ is the radial wavenumber, starting our reference case, 
further scans in this parameter are discussed later. Parallel magnetic fluctuations $\delta B_{\|}$are taken into account and their role will first be discussed.

\subsection{Parallel magnetic fluctuations influence}

The role of the $\delta B_{\|}$has been neglected in many numerical studies of microinstabilities and turbulence in the literature. Here we find that neglecting these effects has a noticeable impact on growth rates even for reasonably small gradients [see Fig.1]. In Fig.1, we show the spectrum of the KBMs obtained by GENE simulations for W7-X with and without $\delta B_{\|}$. For these cases $\beta=3 \%, a / L_{n i, e}=0, a / L_{T i, e}=3$ and $a / L_{T i, e}=7$. The same type of study is then repeated for the tokamak case [see Fig. 2].

The comparison between Fig. 1 and Fig. 2 shows that in both cases an absence of $\delta B_{\|}$ effects leads to a decrease of KBM growth rate up to a factor of 6 . This result is in line with earlier findings that parallel magnetic fluctuations act destabilising $[19,28]$. In the stellarator case, for small gradient $a / L_{T i, e}=3, \delta B_{\|}$is essential for KBMs destabilization [see Fig.1a]. We also note that, for W7-X, the growth rate of the high- $k_{y}$ end of the spectrum (trapped electron modes - TEMs) is not affected by parallel magnetic fluctuations.

For the stellarator, at large gradients, the mode frequency is $\omega_{r}=\omega_{p_{i}} / 2$ only if $\delta B_{\|}$is included [see Fig.1b]. In tokamak case we observe the same trend [see Fig.2b] however, even with $\delta B_{\|}$included, the frequency of the mode is not $\omega_{p_{i}} / 2$ for finite $k_{y}$ s since the temperature gradient $a / L_{T i, e}=7$ is not high enough (for a more detailed discussion about tokamak case see Ref.[17]).

We conclude that local GK KBM simulations are consistent with diamagnetic modification of ideal MHD only if magnetic compressibility is taken into account. 

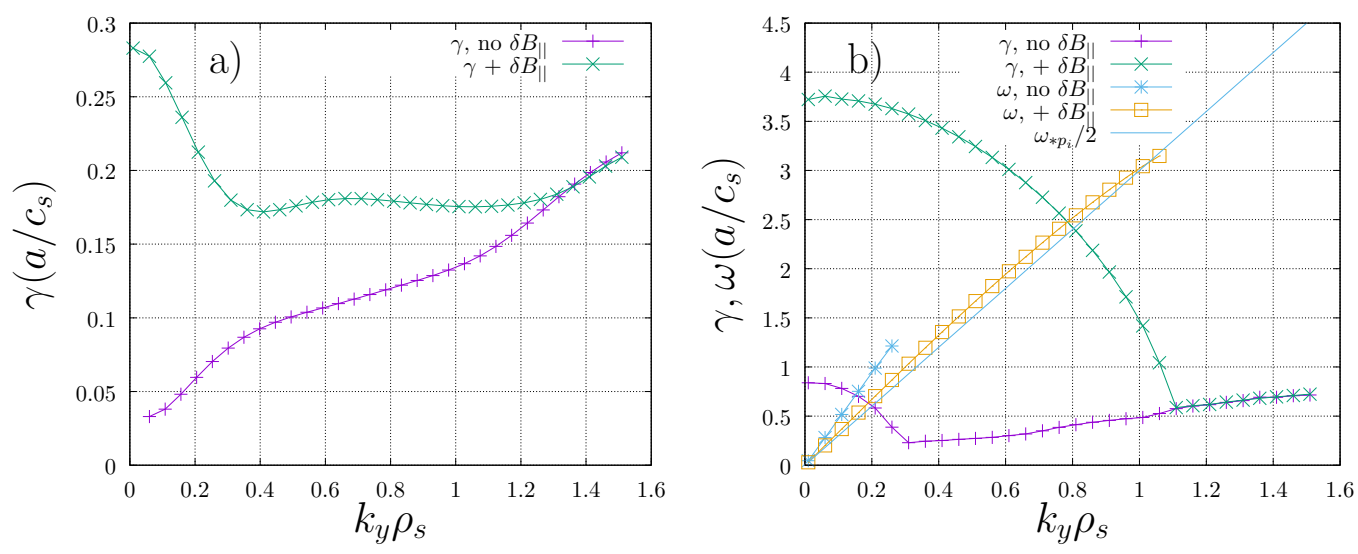

Figure 1: Influence of $\delta B_{\|}$, spectra for W7-X from GENE simulations. Plot $a: a / L_{T i, e}=3$. Plot $b: a / L_{T i, e}=7$.
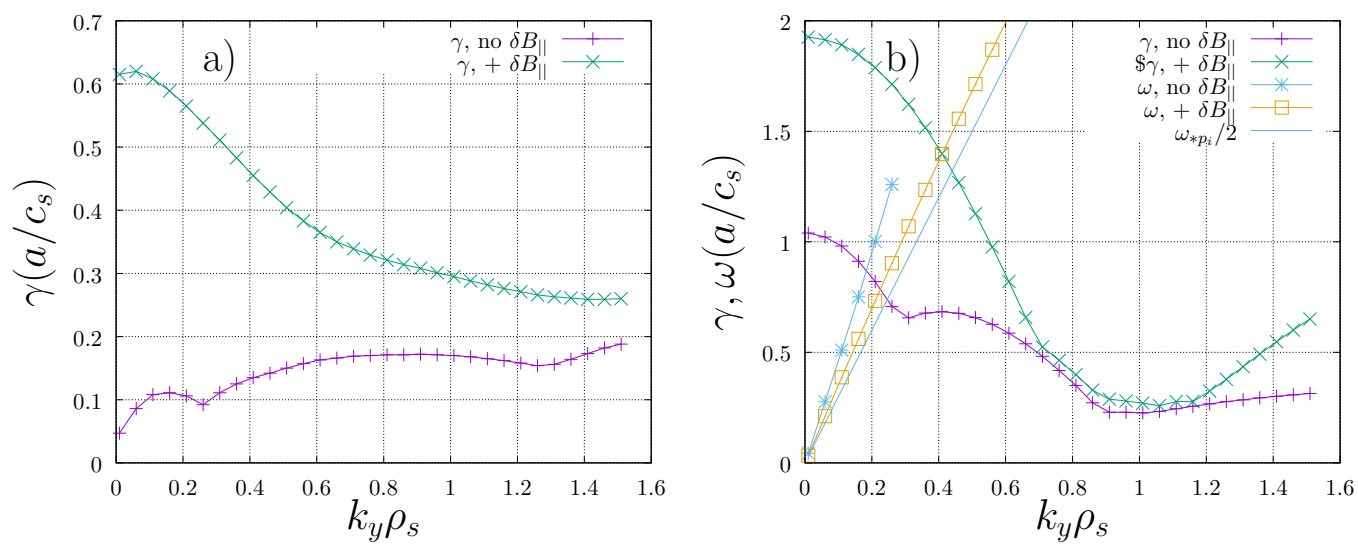

Figure 2: Influence of $\delta B_{\|}$, spectra for tokamak from GENE simulations. Plot $a: a / L_{T i, e}=3$. Plot $b: a / L_{T i, e}=7$.

\subsection{Scan in radial wavenumber}

The most unstable KBM in W7-X geometry has vanishing radial wavenumber (Fig.3a), which corresponds to $\theta_{0}=0$ in the ballooning representation. This is connected with the fact that $k_{x}$ is a function of the global shear, $\hat{s}$, and W7-X has a very small $\hat{s}$. From this it follows the weak dependence of our results on the specific choice of the simulated tube. 
We remark that the radial wavenumber of the most unstable KBM in W7-X is in contrast with that of LHD [13] which is finite. The ITG mode also has the peak of the growth rate curve located around $k_{x} \rho_{s}=0$ (Fig.3b). In calculations shown in this figure, the density gradient and $\beta$ have both been set to zero, so that all microinstabilities other than the ITG mode are eliminated. Periodicity in both plots is explained by the periodic dependence in an envelope of a function which is required for a function in a real space to be periodic [see Eq.4 of Ref.[29]]. In Fig.4 KBM normalized eigenfunction is presented for two simulation tubes. Peaking of eigenfunction around non-zero $\theta$ (see Fig.4, right) is an interesting feature of the simulation tube which is centred around the outboard midplane of the "triangular" plane. Earlier simulations have consistently showed this feature of the eigenfunction being very small at the "helical edge" on the outboard side of W7-X, presumably because the local magnetic shear is exceedingly large there $[30,31]$.
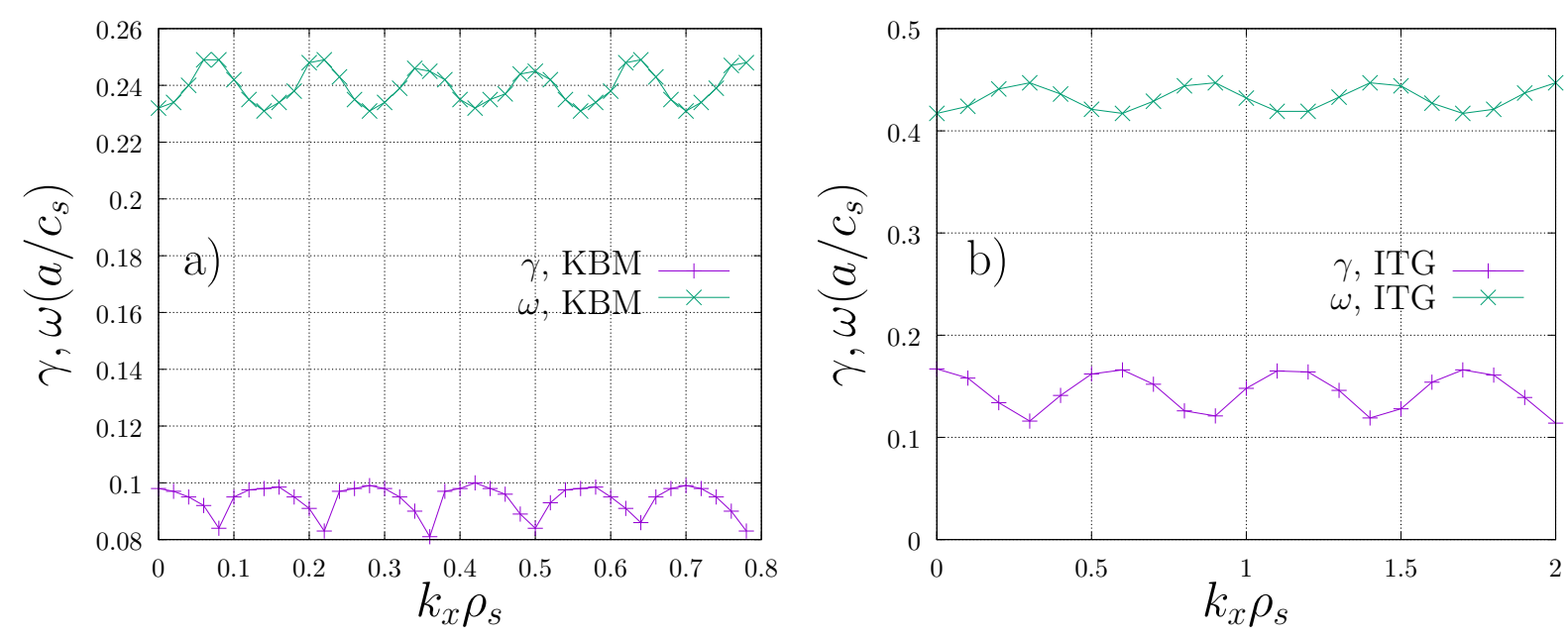

Figure 3: Growth rate (purple, + ) and real frequency (green, $\mathrm{x}$ ) dependence on $k_{x} \rho_{s}$ for a) ITG instability, $a / L_{T i, e}=2, k_{y} \rho_{s}=0.8, \beta=0.0 \%$, and b) KBM instability, $a / L_{T i, e}=2, k_{y} \rho_{s}=0.05$, $\beta=2.4 \%$. Simulation tube is centred around the outboard midplane of the so-called a) "bean" plane and b) "triangular" plane. 

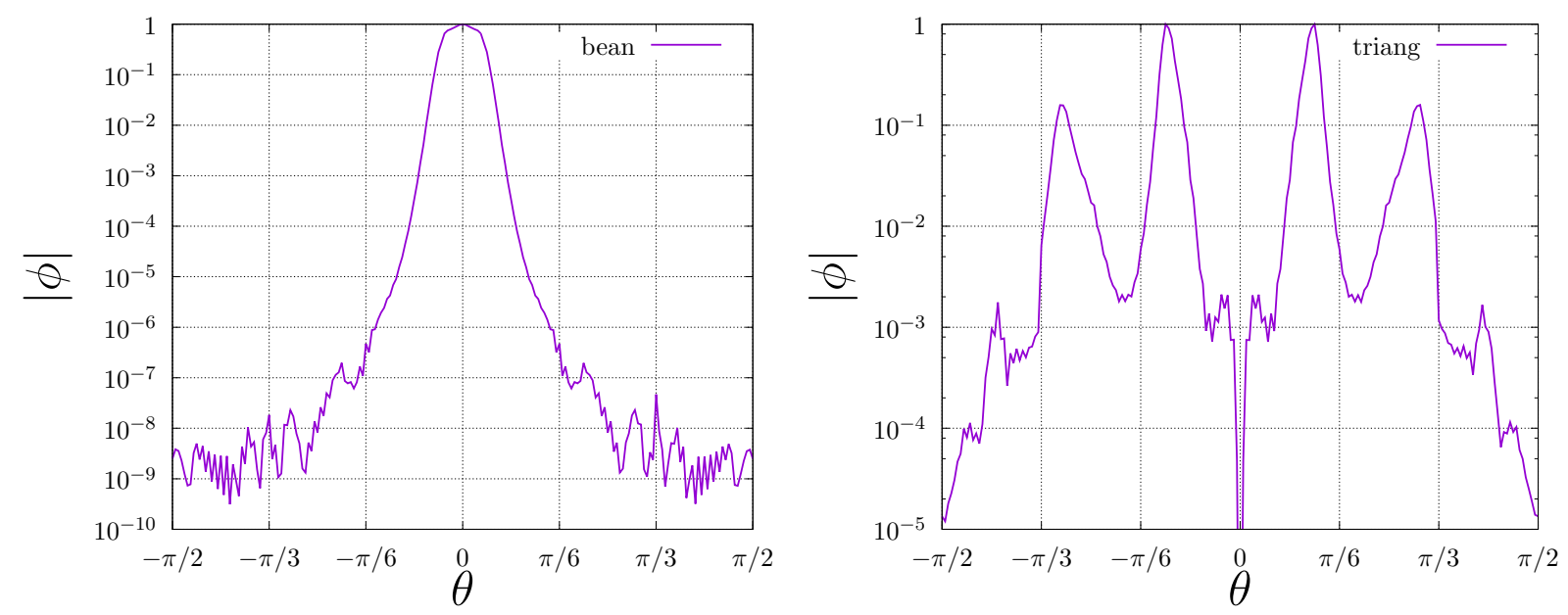

Figure 4: KBM instability eigenfunction, normalized, logarithmic scale. From left to right: simulation tube centred around the outboard midplane of the so-called "bean" plane and around the outboard midplane of the "triangular" plane.

\subsection{The effect of temperature gradients in $\mathrm{W} 7-\mathrm{X}$}

Equation (3) implies that a necessary condition for instability is $\operatorname{Re}(\omega)=\omega_{p_{i}} / 2$. In Ref.[17] it was shown that this is indeed observed in numerical simulations for large temperature gradients for a simple tokamak geometry. We found that the same is true in the stellarator W7-X [see Fig.5]. This simulation corresponds to an MHD mode in the limit $k_{y} \rightarrow 0$, since on this plot we can observe a purely growing mode with maximum of growth rate at $k_{y}=0$. Exactly the same type of instability in simple tokamak geometry was discussed in [17]. 


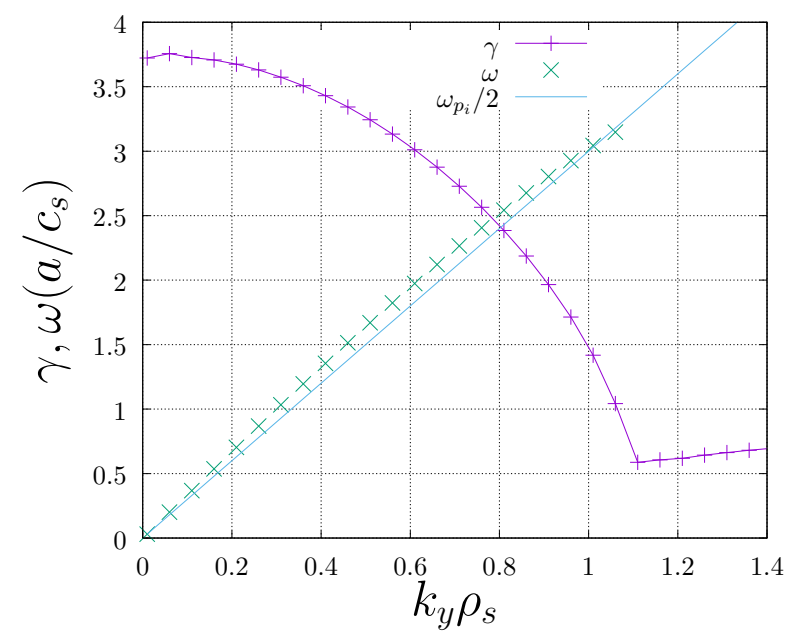

Figure 5: Spectrum for $a / L_{T i, e}=7$. Here $\beta=3 \%$ and $a / L_{n i, e}=0 . \gamma$ : solid curve $(+)$ and $\omega_{r}$ : crosses $(\times)$. The blue line represents the $\omega_{p i} / 2$ frequency.
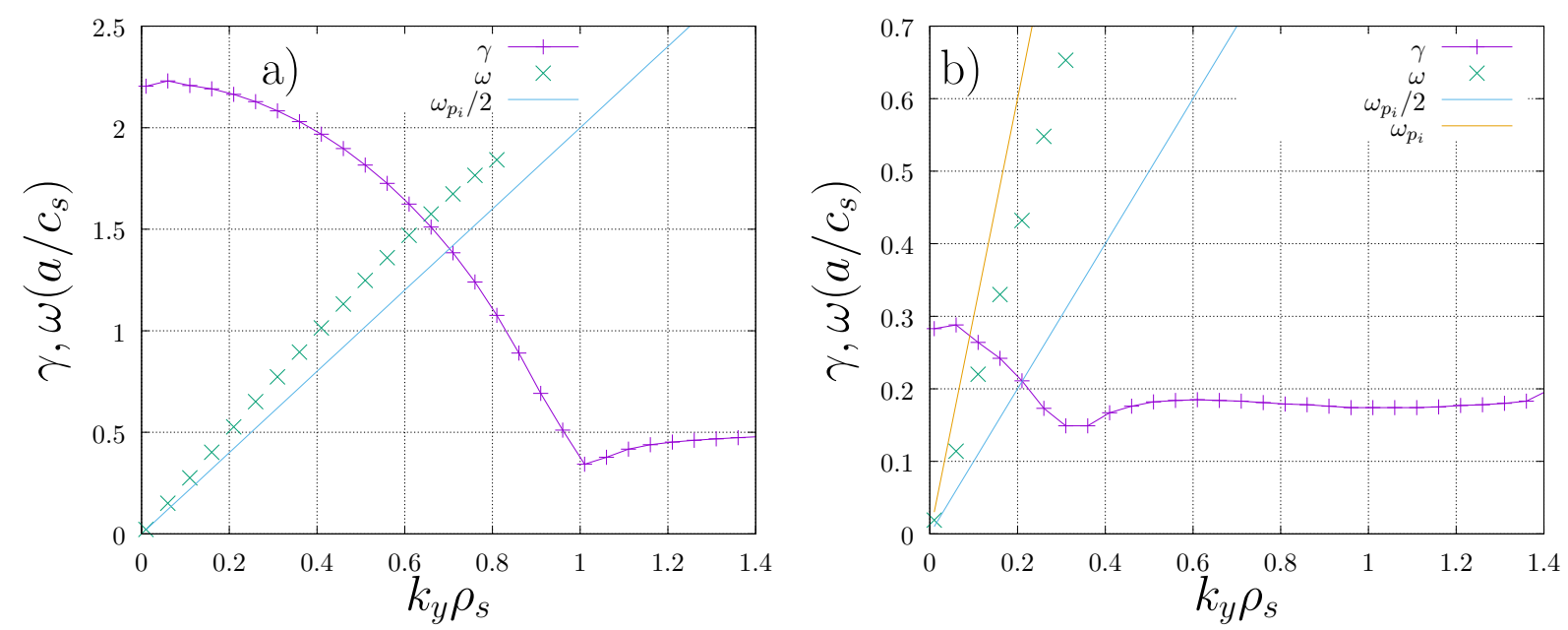

Figure 6: Spectra for a) $a / L_{T i, e}=5$ and for b) $a / L_{T i, e}=3$. Here $\beta=3 \%$ and $a / L_{n i, e}=0 . \gamma$ : solid curve $(+)$ and $\omega_{r}$ : crosses $(\times)$. The blue line represents the $\omega_{p i} / 2$ frequency, orange one represents the $\omega_{p i}$ frequency.

In $\mathrm{W} 7-\mathrm{X}$ we find that KBMs have zero frequency at $k_{y}=0$ for a range of temperature gradients [see Fig.6]. Another important aspect is that, despite gradients being so small that $\omega_{r}$ deviates substantially from $\omega_{p i} / 2$, yet the growth rate of the mode peaks at $k_{y}=0$, as opposed to the tokamak, in which an increase in frequency is accompanied by a peaking 
of the growth rate at finite wavelengths.

W7-X was optimized such away that most magnetic configurations have a high threshold for ideal-MHD instability [32]. It is therefore important to establish whether kinetic effects can destabilize ballooning modes for $\beta$ s lower than the ideal ones. In the next subsection we investigate this question.

\subsection{KBM and ideal MHD thresholds in W7-X}

Results of GENE simulations for two W7-X configurations are shown in Fig.7. One of these configurations (TEH, purple color, + ) is atypical (non-optimised) in the sense that it has no magnetic well and is therefore MHD-unstable for very low $\beta$, whereas the other configuration (KJM, green, $\mathrm{x}$ ) is a typical one and is MHD-stable up to $\langle\beta\rangle=5 \%$ (where $\langle\beta\rangle$ is a plasma volume average $\beta$, not to be confused with local $\beta$ which is used in the present work). Growth rates (a) and real frequencies (b) are displayed as functions of $\beta$. For these calculations we used zero density gradient, $a / L_{T i, e}=2$, and $k_{y} \rho_{s}$ was varied from 0.05 to 0.8 to identify the maxima of growth rates. The TEH configuration is characterised by a small rotational transform, low iota $<5 / 6$, and very large mirror ratio $=24 \%$, whereas the KJM configuration corresponds to a more typical case with mirror ratio $=10 \%$ and standard iota $=1$. 

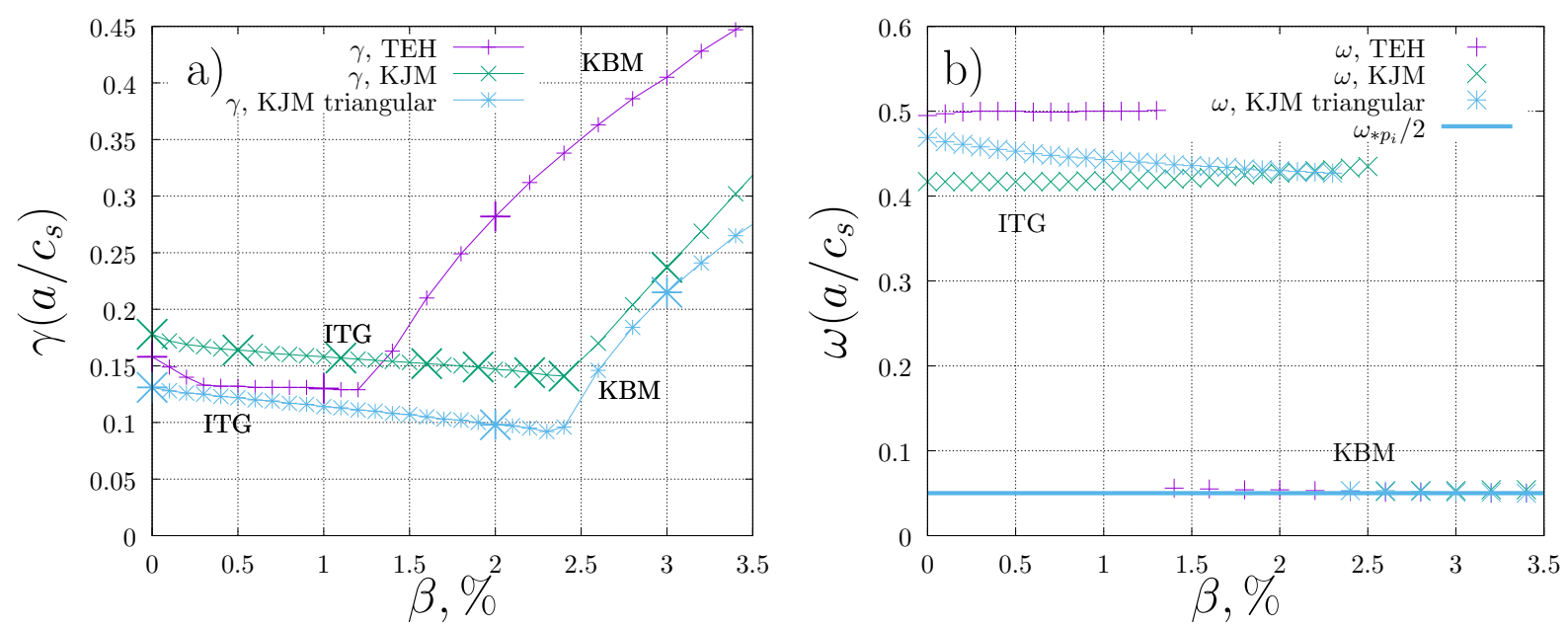

Figure 7: Dependence on $\beta$ for the fastest growing mode with $a / L_{T i, e}=2$ and $k_{y} \rho_{s}$ in the range from 0.05 to 0.8 , in two different W7-X configurations (KJM and TEH) and two simulation tubes (centred around the outboard midplane of the "bean" and "triangular" plane). (a): growth rate of the instabilities. (b): real frequency of the corresponding modes. Large dots specify where the magnetic equilibrium was varied.

We see that the "diamagnetically-modified ideal MHD" ballooning mode description (based on our $\omega_{r}$ condition, blue line Fig.7b) is valid for all KBMs presented on this plot. The extrapolation of growth rate to where the KBM branch ot the curves in Fig.7 would intersect the horizontal axis, $\gamma=0$, suggests that the critical beta of KBM destabilization in the MHD non-optimised configuration is $\beta \sim 0.65 \%$ while, for MHD optimised ones it is $\beta \sim 1.9 \%$ ("bean" tube) and $\beta \sim 2.2 \%$ ("triangular" tube). We conclude that the MHD-optimized configuration has a higher threshold for KBMs. This result confirms the expectation that KBM stability should be related to ideal-MHD stability. A device like W7-X that has been optimised to be MHD-stable up to very high $\beta$ should therefore also benefit from enhanced KBM stability. However, in previous simulations it has typically been seen that the KBM stability threshold lies somewhat below that of ideal MHD ballooning modes. In Fig.8 we show KBM critical $\beta$ values (for KJM and TEH configurations) corresponding to the point of marginal KBM stability: for $k_{y} \rho_{s}$ from 0.005 to 0.4 and a range of $\beta$ values, the linear growth rates were obtained and KBM curves were extrapolated to a subdominant region 
where KBMs are marginally stable (the same procedure was described in Ref.[35] for $s-\alpha$ tokamak geometry). Reference ideal MHD $\beta_{\text {crit }}$ values (note, $\beta_{\text {crit }}$ is a standard local one) for both configurations were obtained by using a separate code [34] which solves the ideal MHD ballooning equation along the field line. Note that the kinetic threshold is determined at the specific $k_{y}$ where the KBM is destabilized first, which, in the TEH case is close to $k_{y} \approx 0$, as it should in the ideal MHD (iMHD) limit. In TEH case, a higher stability limit of KBMs [as compared with the ideal MHD limit], comes from kinetic effects, for example diamagnetic [see eq. Eq. (3)]. However, in the KJM case, we see a KBM threshold significantly lower than the corresponding iMHD limit. This might be connected with further kinetic effects, which can provide additional destabilization close to marginality. One could perhaps attribute it to the resonant ion-transit destabilization [for more details see Ref.[36]]. A numerical study of KBMs observed above and below the iMHD limit was also performed in Ref. [35] for $s-\alpha$ tokamak geometry.

Thus we conclude that, even though W7-X has been optimised to be MHD-stable up to very high $\beta$ and indeed the MHD-optimized configuration has a relatively high threshold for KBMs, kinetic effects are important and we may expect KBM instability before we reach the iMHD ballooning threshold.

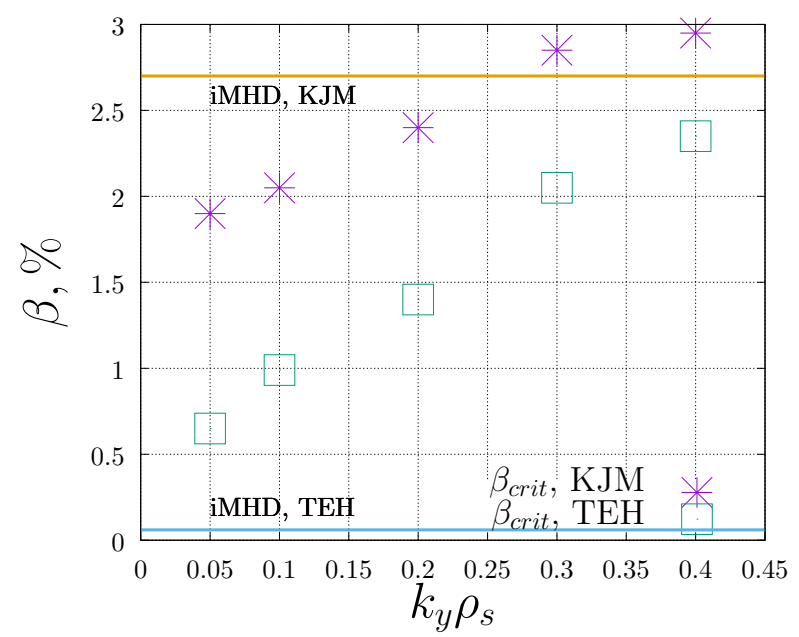

Figure 8: KBM critical $\beta$ values corresponding to the point of marginal KBM stability (see the text). For reference: the MHD estimates obtained by an ideal ballooning code are shown as lines. 


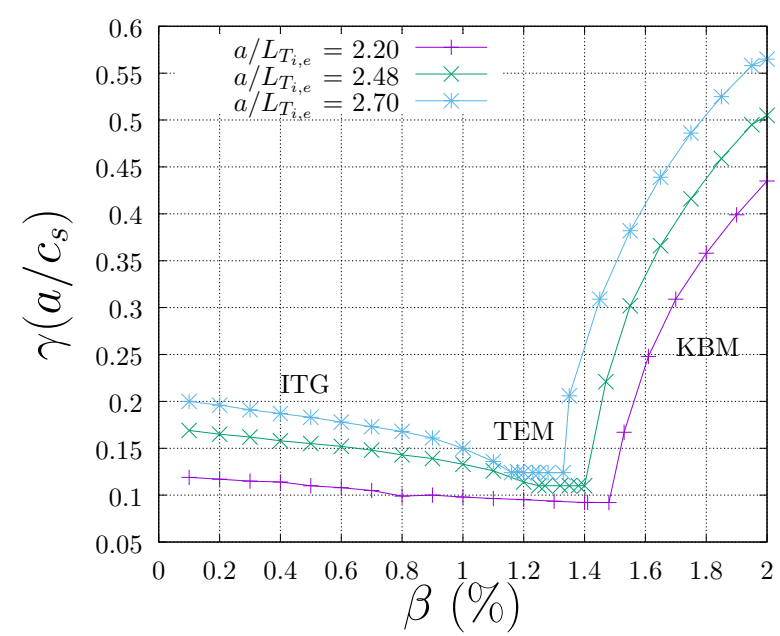

Figure 9: Local flux-tube GENE simulations of the growth rate dependence on plasma $\beta$ for different $a / L_{T i, e}$, standard Cyclone Base Case parameters, collisionless regime, $\hat{s}-\alpha$ geometry.

In Fig.7, the growth rate of ITG instability decreases moderately with $\beta$ and the ITG mode is subdominant for $\beta>2.4 \%$ in the MHD-optimized case (KJM) and for $\beta>1.2 \%$ in the MHD-non-optimized one (TEH). However, we do not observe regions where instability is completely suppressed between ITG and KBM. A trapped-electron mode (TEM) window is not observed between KBMs and ITGs either (in contrast to the usual picture of the tokamak case, Fig.1 of Ref.[8]) and Fig. 9 of this article). This might be connected with, first, more unstable ITGs (less stabilized by $\beta$ ) and, second - TEMs in W7-X are more stable given the fact that most trapped electrons have favourable bounce-averaged curvature [37]. The same was observed in [13], where the authors reported that the trapped electron mode is not observed in the same type of calculations for the standard Large Helical Device (LHD) case. This is a major difference between electromagnetic microinstabilities in stellarators and tokamaks.

We further proceed with a comparison of the W7-X results with a generic tokamak case, emphasising the consistency of the local pressure gradient and the plasma equilibrium. In Fig. 10 we show the growth rates obtained from the gyrokinetic calculations performed by GENE code as functions of $\beta$ and $k_{y}$. 

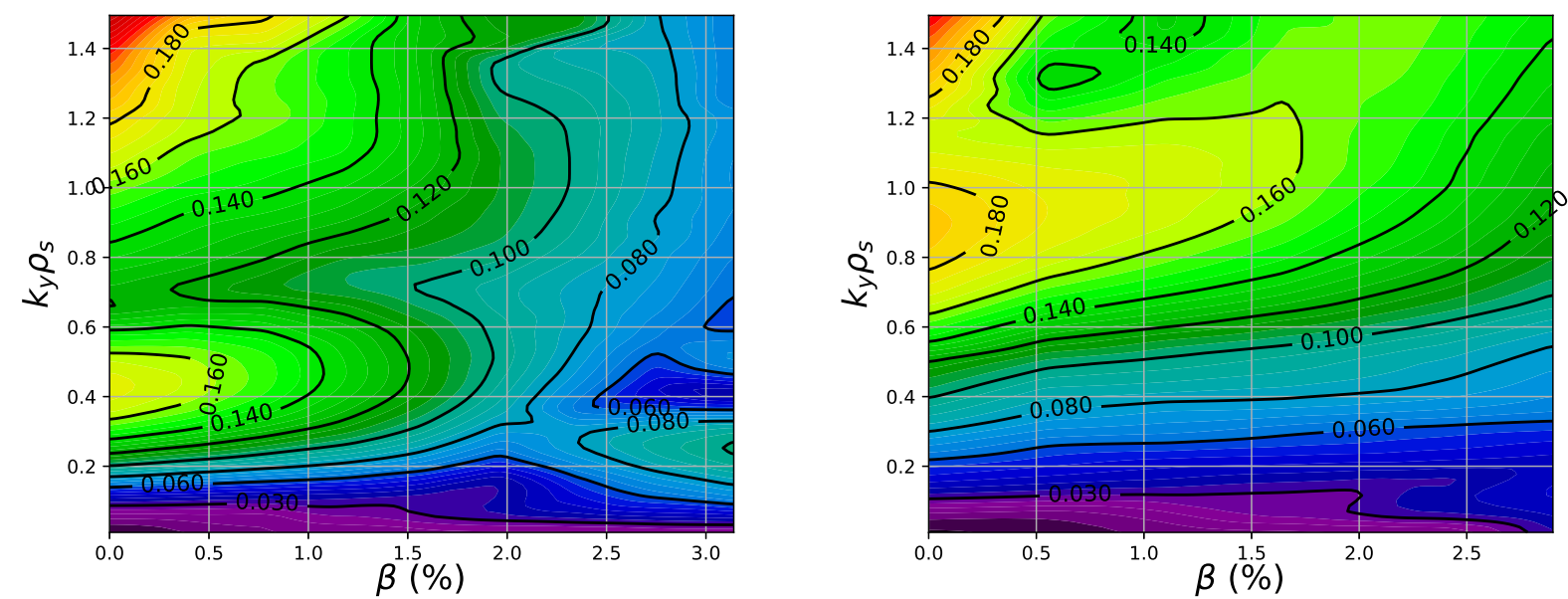

Figure 10: Growth rates as a function of $\beta$ and $k_{y} \rho_{s}$ (colorcoded), when pressure gradients are consistent with the plasma equilibrium. Left: simulations for standard tokamak case. Right: W7-X standard configuration (KJM, "bean" tube).

For these calculations we include $\delta B_{\|}$effects (as we do in all our calculations) and take pressure gradients in GENE to be consistent with the plasma equilibrium obtained by the VMEC code [26]. We stress the importance of this type of consistency because the difference with results obtained in a non-consistent way (see Fig. 11) is evident. In Fig. 10 we can observe different types of electromagnetic microinstabilities. For the tokamak standard case ( Fig. 10, left plot) the region with $\beta<1.7 \%$ and $k_{y} \rho_{s}<0.7$ shows electromagnetic ITG modes, while the region with $\beta>2.3 \%$ and $0.1<k_{y} \rho_{s}<0.4$ contains KBMs. TEMs can be observed in the region with $k_{y} \rho_{s}>0.8$. For the W7-X standard case ( Fig. 10, right plot) ITG instability exists in a wider region of parametrs, and complete electromagnetic stabilization of this instability does nor appear. KBMs are more stable than in the tokamak case and the most unstable ones are those with the smallest wavenumbers. TEMs are present for high $k_{y}$ but cannot be observed in the region between ITG and KBM (as has been previously discussed). We conclude this W7-X configuration appears to be somewhat more stable than the tokamak with respect to low $k_{y}$ modes, including KBMs.

As already mentioned, simulations with consistent plasma equilibria are quite different from those without. 

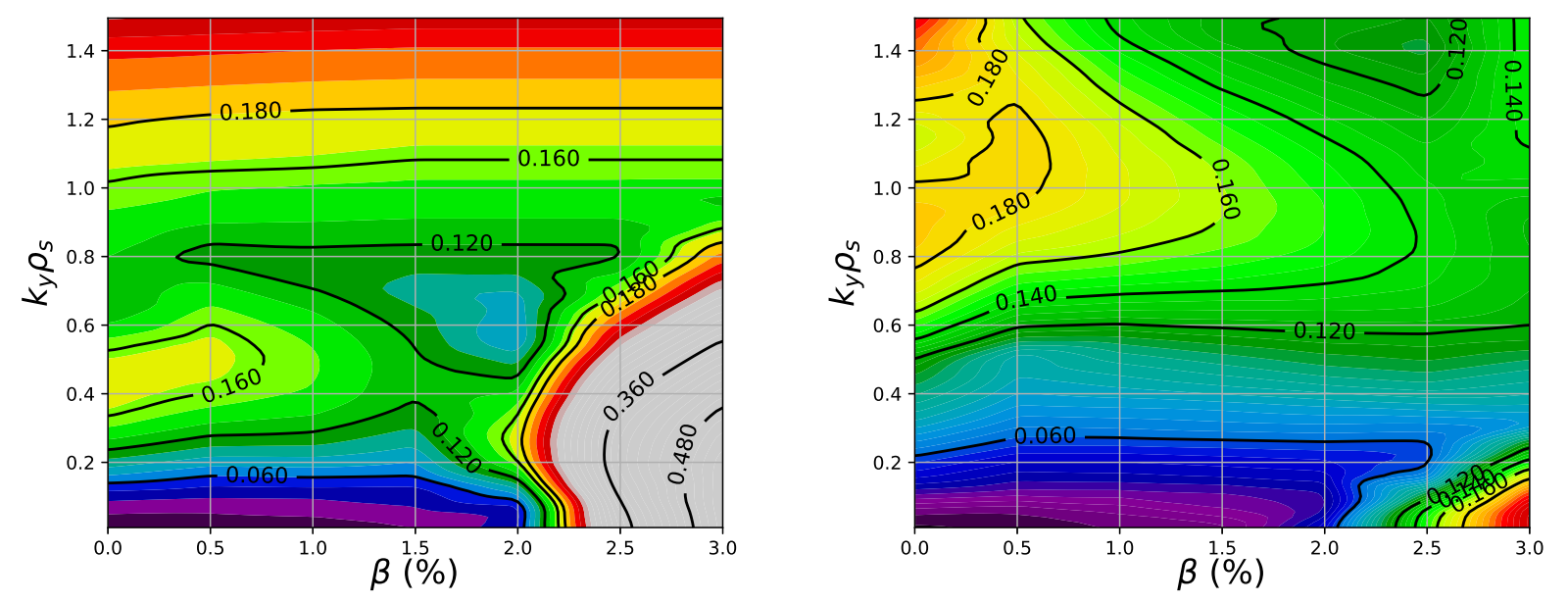

Figure 11: Growth rates as a function of $\beta$ and $k_{y} \rho_{s}$ (colorcoded), when pressure gradients are not (!) consistent with the plasma equilibrium. Left: simulations for standard tokamak case. Right: W7-X standard configuration (KJM, "bean" tube).

In Fig.11 we show the result of simulations in a fixed equilibrium (zero $\beta$ ) that does not vary with $\beta$ and observe that growth rates for ITG and TEM for small values of $\beta$ are approximately the same (in comparison with Fig.10) but KBMs are significantly more unstable at higher values of $\beta$, both in the tokamak and in W7-X. However, in the stellarator case, they share the same feature of having the most unstable mode around $k_{y} \rho_{s}=0$. The most interesting point here is that the TEM for the tokamak case seems not to be affected by $\beta$ if the equilibrium is kept constant, but if it is varied consistently with the pressure gradients (Fig. 10) the TEM is stabilized by $\beta$ as originally predicted by Rosenbluth and Sloan [38]. For our W7-X calculations the TEM is moderately stabilized by $\beta$ in both cases, both with consistent and non-consistent equilibria.

\section{CONCLUSION}

In the present work, linear electromagnetic gyrokinetic numerical simulations of microinstabilities have been performed with the GENE code in finite- $\beta$ plasmas for different geometries in the stellarator device Wendelstein $7-\mathrm{X}$ as well as a generic tokamak model. 
The results exhibit good agreement of the real frequencies of KBMs with the diamagnetic modification of ideal MHD limit in the large-gradient-regime for W7-X geometry. This finding agrees with a recent KBM study in simple tokamak geometry [17].

Thresholds for KBM mode destabilization in W7-X configurations with different ideal MHD stability properties have been compared and found to be correlated. Simulations of the KBM instability in the standard (MHD-optimized) W7-X geometry with $\beta=3 \%$, flat density profile and temperature gradient $a / L_{T i, e}=2$ show that the KBM stability threshold $\left(k_{y} \rho_{s}=0.05\right)$ is about $2.2 \%$ (simulation tube centred around the outboard midplane of the "triangular" plane) and $1.9 \%$ ("bean") as compared with $0.65 \%$ in the non-optimized configuration, which has a much lower MHD stability threshold. KBM critical $\beta$ values correspond to the point of marginal KBM stability were compared with the reference MHD estimates obtained by ideal ballooning code and were found to be lower in KJM case. Thus we conclude that we may expect a rise of KBM instability before corresponding iMHD threshold.

The comparison of W7-X and a generic tokamak shows that one of the significant features of the KBM in W7-X geometry is that the most unstable mode has $k_{y} \rho_{s}=0$ for a wide range of simulation parameters. This is in contrast to the tokamak configuration, where the most unstable mode closer to the marginality has a finite $k_{y} \rho_{s}$. In W7-X, the ITG mode is progressively suppressed with increasing $\beta$ but is not stabilized completely and the critical value for the onset of KBM is about $\beta=2.5 \%$ for $a / L_{T i, e}=2$, flat density gradient and and $\hat{s} \approx-0.1$. For the tokamak case, we observe a more significant $\beta$ stabilization of the ITG mode and more unstable KBMs $(\beta=2.1 \%)$. We conclude that this W7-X configuration appears to be more stable than the tokamak case with respect to low $k_{y}$ modes, including KBMs.

In all these simulations, it is important to keep the magnetic equilibrium geometry consistent with the varying pressure gradient in the gyrokinetic simulations. This is clearly seen when we compare our results with simulations obtained without this sort of concordance. It is noted that $\beta$ stabilization of TEM in the tokamak case appears only if the equilibrium is varied consistently with the pressure gradient, whereas in W7-X calculations, the TEM is also (moderately) stabilized by $\beta$ even if the equilibrium is kept fixed. 


\section{Acknowledgements}

The authors are grateful to F. Zonca and F. Jenko for for constructive comments and fruitful discussions and Y. Turkin for technical support. GENE simulations were performed at Computer Centre in Garching.

[1] A. Zocco, P. Xanthopoulos, H. Doerk, J. W. Connor, J. Plasma Phys., vol. 84, 715840101 (2018)

[2] V. Kornilov, R. Kleiber, R. Hatzky, L. Villard and G. Jost, Phys. Plasmas 113196 (2004)

[3] P. Xanthopoulos, F. Merz, T. Gorler, F. Jenko, PRL 99, 035002 (2007)

[4] J. Riemann, R. Kleiber and M. Borchardt, Plasma Physics and Controlled Fusion, 58 (7) (2016)

[5] P. Xanthopoulos, Phys. Plasmas 14, 042501 (2007)

[6] J.H.E. Proll, H.E. Mynick, P. Xanthopoulos, S.A. Lazerson, B.J. Faber, Plasma Physics and Controlled Fusion 58 (1), 014006 (2015)

[7] J.H.E. Proll, P. Helander and Xanthopoulos, Physics of Plasmas 20122506 (2013)

[8] M. J. Pueschel, M. Kammerer, and F. Jenko, Phys. Plasmas 15, 102310 (2008)

[9] M. Kotschenreuther, Phys. Fluids 29, 2898 (1986)

[10] J. A. Baumgaertel, E. A. Belli, W. Dorland, W. Guttenfelder, G. W. Hammett, D.R. Mikkelsen, G. Rewoldt, W.M. Tang, P. Xanthopoulos, Phys. Plasmas 18, 122301 (2011)

[11] A. Mishchenko, R. Hatzky, A. Könies, Phys. Plasmas 15 (11), 112106, (2008)

[12] A. Biancalani, A. Bottino, S. Briguglio, A. Könies, Ph. Lauber, A. Mishchenko, E. Poli, B. D. Scott and F. Zonca, Phys. Plasmas 23, 012108 (2016)

[13] A. Ishizawa, S. Maeyama, T.-H. Watanabe, H. Sugama and N. Nakajima, J. Plasma Phys. $81,435810203(2015)$

[14] A. Mishchenko, M. Borchardt, M. Cole, R. Hatzky, T. Fehér, R. Kleiber, A. Kon̈ies and A. Zocco, Nucl. Fusion 55 (5), 053006 (2015)

[15] R.J. Hastie and K.W. Hesketh, Nucl. Fusion 21651 (1981)

[16] W.M. Tang, J.W. Connor, and R. J. Hastie, Nucl. Fusion 20, 1439 (1980)

[17] K. Aleynikova and A. Zocco, Physics of Plasmas 24, 092106 (2017) 
[18] J.W. Connor, R.J. Hastie, and J.B. Taylor, Phys. Rev. Lett. 40 (1978)

[19] A. Zocco, P. Helander, J.W. Connor, Plasma Physics and Controlled Fusion 57 (8) (2015)

[20] F. Jenko, W. Dorland, M. Kotschenreuther, and B.N. Rogers, Phys. Plasmas 7, 1904 (2000)

[21] T. Dannert and F. Jenko, Phys. Plasmas 12, 72309 (2005)

[22] M. Kotschenreuther, G. Rewoldt, and W.M. Tang, Comput. Phys. Commun. 88, 128 (1995)

[23] W. Dorland, F. Jenko, M. Kotschenreuther, and B.N. Rogers, Phys. Rev. Lett. 85, 5579 (2000)

[24] K.V. Roberts and J.B. Taylor, Phys. Rev. Lett. 8, 197 (1962)

[25] A. H. Boozer, Phys. Fluids 23, 2283 (1980)

[26] S.P. Hirshman, W.I. van Rij, and P. Merkel, Comput. Phys. Commun. 43, 143 (1986)

[27] P. Xanthopoulos, W.A. Cooper, F. Jenko, Y. Turkin, A. Runov, J. Geiger, Phys. Plasmas 16, $082303(2009)$

[28] E. A. Belli and J. Candy, Phys. Plasmas 17, 112314 (2010)

[29] D. Dickinson, C. M. Roach, J. M. Skipp, H. R. Wilson, Phys. Plasmas 21, 010702 (2014)

[30] R. Kleiber and B. Scott, Phys. Plasmas 12102507 (2005)

[31] P. Helander, C. D. Beidler, T. M. Bird, M. Drevlak, Y. Feng, R. Hatzky, F. Jenko, R. Kleiber, J. H. E. Proll, Yu. Turkin, and P. Xanthopoulos, Plasma Phys. Controlled Fusion 54, 124009 (2012)

[32] C. Nührenberg, Nucl. Fusion 56076010 (2016)

[33] A.M. Dimits, G. Bateman, M.A. Beer, B.I. Cohen, W. Dorland, G.W. Hammett, C. Kim, J.E. Kinsey, M. Kotschenreuther, A.H. Kritz, L.L. Lao, J. Mandrekas, W.M. Nevins, S.E. Parker, A.J. Redd, D.E. Shumaker, R. Sydora, and J. Weiland, Phys. Plasmas 7, 969 (2000)

[34] J. Nuehrenberg and R. Zille, Equilibrium and stability of low-shear stellarators, Theory of fusion plasmas 1987, (Società Italiana di Fisica, Bologna, ISBN 88-7794-009-3), 1988, p3.A

[35] M. J. Pueschel and F. Jenko, Phys. Plasmas 17, 062307 (2010)

[36] S.T. Tsai, L. Chen, Phys. Fluids B 5, 3284 (1993)

[37] J.H.E. Proll, P. Helander, G. G. Plunk, J.W. Connor, Phys. Rev. Lett. 108245002 (2012)

[38] M. Rosenbluth, M. L. Sloan, Physics of Fluids 14, 1725 (1971)

[39] G. Rewoldt, L.-P. Ku, and W. M. Tang, Physics of Plasmas 12, 102512 (2005) 